\title{
Testing surface area with arbitrary accuracy
}

\author{
Joe Neeman \\ Department of Electrical and Computer Engineering, and \\ Department of Math \\ University of Texas at Austin \\ joeneeman@gmail.com
}

\begin{abstract}
Recently, Kothari et al. gave an algorithm for testing the surface area of an arbitrary set $A \subset[0,1]^{n}$. Specifically, they gave a randomized algorithm such that if $A$ 's surface area is less than $S$ then the algorithm will accept with high probability, and if the algorithm accepts with high probability then there is some perturbation of $A$ with surface area at most $\kappa_{n} S$. Here, $\kappa_{n}$ is a dimension-dependent constant which is strictly larger than 1 if $n \geq 2$, and grows to $4 / \pi$ as $n \rightarrow \infty$.
\end{abstract}

We give an improved analysis of Kothari et al.'s algorithm. In doing so, we replace the constant $\kappa_{n}$ with $1+\eta$ for $\eta>0$ arbitrary. We also extend the algorithm to more general measures on Riemannian manifolds.

\section{Categories and Subject Descriptors}

Mathematics of Computing [Probability and Statistics]: Probabilistic Algorithms

; Theory of Computation [Randomness, Geometry, and Discrete Structures]: Computational Geometry

\section{General Terms}

surface area, noise sensitivity, property testing

\section{INTRODUCTION}

Consider the problem of estimating the surface area of a set $A \subset \mathbb{R}^{n}$. This fundamental geometric problem has been widely studied, thanks in part to its applications in computer graphics (see, e.g., [14]) and medical imaging (see, e.g., [1, 7]). Due to its diverse applications, surface area estimation has been studied under several different models. In this work, we consider a query access model, in which one can choose points in $\mathbb{R}^{n}$ and ask whether then belong to $A$; this is perhaps the most natural model for applications to learning. In contrast, in stereography 1 one considers the problem of estimating $A$ 's surface area given complete access to many random projections (or slices) of $A$; this is a natural model for certain problems in $3 \mathrm{D}$ imaging.

Testing surface area. Kearns and Ron [9] introduced the study of surface area in the property testing framework. In this framework, which goes back to [6], we fix some predicate $P$ and ask for an algorithm that can distinguish (with high probability) problem instances satisfying $P$ from instances that are far from satisfying $P$. For the specific case of testing the surface area of a set $A \subset[0,1]^{n}$, we denote the Lebesgue measure by $\lambda_{n}$, its corresponding surface area measure by $\lambda_{n}^{+}$, and we ask for an algorithm that accepts if $\lambda_{n}^{+}(A) \leq S$ and rejects if $\lambda_{n}^{+}(B) \geq(1+\eta) S$ for all $B$ satisfying $\lambda(B \Delta A) \leq \epsilon$. This problem was first considered by Kearns and Ron 9 in the case $n=1$. They showed that it can be solved for any $\epsilon>0$ with $O(1 / \epsilon)$ queries, provided that $\eta \geq 1 / \epsilon$. The approximation error was improved by Balcan et al. 4, who gave an algorithm (still for $n=1$ ) that requires $O\left(\epsilon^{-4}\right)$ queries but works for $\eta=0$. Substantial progress was made by Kothari et al. 11], who gave an algorithm that works in arbitary dimensions, but requires $\eta$ to be bounded away from zero as soon as $n \geq 2$.

Our main result is an improvement of Kothari et al.'s analysis, showing that for any $\epsilon, \eta>0$, an essentially identical algorithm requires $O\left(\eta^{-3} \epsilon^{-1}\right)$ samples, and guarantees that if the algorithm rejects with substantial probability then $\lambda_{n}^{+}(B) \geq(1+\eta) S$ for all $B$ satisfying $\lambda(B \Delta A) \leq \epsilon$. Besides the improvement in Kothari et al.'s soundness condition, our other main contribution is a unified argument that applies to more general spaces than $[0,1]^{n}$ with the Lebesgue measure.

Other work on estimating surface area. The property testing formulation of surface area measurement asks for fairly weak guarantees compared to the more general problem of estimating the surface area of $A$. One advantage of these weaker requirements is that the problem can be solved in much greater generality, and with fewer queries. For example, the state of the art in surface area estimation for convex sets is an algorithm of Belkin et al. 5] which requires $\tilde{O}\left(n^{4} \epsilon^{-2}\right)$ queries. One obvious difficulty in moving beyond convex sets is that a general set $A$ may have large surface area, but only because of some very small part of it in which the boundary wiggles crazily. Cuevas et al. 7 dealt with this issue by imposing some regularity on the boundary of $A$, but their algorithm required $O\left(\epsilon^{-2 n}\right)$ samples. 
The property testing framework deals with the "crazy boundary" issue in quite a simple way. Essentially, we relax the soundness condition so that if $A$ can be modified on a set of measure $\epsilon$ in order to reduce its surface area below $(1+\eta) S$, then we allow the algorithm to accept.

Surface area and learning. One application for surface area testing - which was noted already by Kothari et al. is in the "testing before learning" framework. Indeed, Klivans et al. 10] gave an algorithm for agnostically learning sets in $\mathbb{R}^{n}$ under the Gaussian measure; their algorithm runs in time $n^{O\left(S^{2}\right)}$, where $S$ is the Gaussian surface area of the set that they are trying to learn. In particular, if we could test the Gaussian surface area of a set before trying to learn it, we would have advance notice of the learning task's complexity. (Although we have not yet mentioned Gaussian surface area, our results hold just as well in that case as they do for Lebesgue surface area.)

Surface area and noise sensitivity. Consider the torus $\mathbb{T}^{n}=(\mathbb{R} / \mathbb{Z})^{n}$ with the Lebesgue measure $\lambda_{n}$ (from now on, for technical convenience, we will consider sets $A \subset \mathbb{T}^{n}$ instead of sets $\left.A \subset[0,1]^{n}\right)$. Let $X$ be a uniformly distributed point in $\mathbb{T}^{n}$ and set $Y=X+\sqrt{2 t} Z$, where $Z \sim \mathcal{N}\left(0, I_{n}\right)$ is a standard Gaussian vector. For a set $A \subset \mathbb{T}^{n}$, we define the noise sensitivity of $A$ at scale $t$ by

$$
\mathrm{NS}_{t}(A)=\operatorname{Pr}(X \in A, Y \notin A)+\operatorname{Pr}(Y \in A, X \notin A) .
$$

Crofton, inspired by the Comte de Buffon's famous needle problem, was the first to make a connection between surface area and noise sensitivity. His classical formula (see, e.g., [15]) implies that if $A \subset \mathbb{T}^{n}$ is a set with $\mathcal{C}^{1}$ boundary then the surface area of $A$ is equal to $\frac{2 \sqrt{t}}{\sqrt{\pi}}$ times the expected number of times that the line segment $[X, Y]$ crosses $\partial A$. Since this number of crossings is always at least 1 on the event $\left\{1_{A}(X) \neq 1_{A}(Y)\right\}$, we have the inequality

$$
\mathrm{NS}_{t}(A) \leq \frac{2 \sqrt{t}}{\sqrt{\pi}} \lambda_{n}^{+}(A),
$$

where $\lambda_{n}^{+}$denotes the surface area.

The inequality (11) cannot be reversed in general. To construct a counter-example, note that $A$ may be modified on a set of arbitrarily small measure (which will affect the left hand side of (1) by an arbitrarily small amount) while increasing its surface area by an arbitrarily large amount. The main geometric result of this work is that when $t$ is small, these counter-examples to a reversal of (10) are essentially the only ones possible.

TheOREM 1.1. For any $A \subset \mathbb{T}^{n}$ with $\mathcal{C}^{1}$ boundary, and for every $\eta, t>0$, there is a set $B \subset \mathbb{T}^{n}$ with $\lambda_{n}(A \Delta B) \leq$ $\mathrm{NS}_{t}(A) / \eta$ and

$$
\frac{2 \sqrt{t}}{\sqrt{\pi}} \lambda_{n}^{+}(B) \leq(1+o(\eta)) \mathrm{NS}_{t}(A)
$$

REMARK 1.2. One might wonder whether the $(1+o(\eta))$ term on the right hand side of Theorem 1.1 may be removed. Although we cannot rule out some improvement of Theorem 1.1, one should keep in mind the example of the "dashed-line" set $A_{t} \subset \mathbb{T}^{1}$ consisting of $\lfloor 5 / \sqrt{t}\rfloor$ intervals of length $\sqrt{t} / 10$, separated by intervals of length $\sqrt{t} / 10$. Then $\mathrm{NS}_{t}\left(A_{t}\right)$ is a constant factor smaller than $2 \sqrt{t} / \lambda_{1}^{+}\left(A_{t}\right) / \sqrt{\pi}$ as $t \rightarrow 0$. Moreover, reducing $\lambda_{1}^{+}\left(A_{t}\right)$ by a constant factor would require changing $A_{t}$ on a set of constant measure. In other words, it is not possible to simultaneously have $\lambda_{1}^{+}\left(A_{t} \Delta B\right)=o(1)$ and

$$
\frac{2 \sqrt{t}}{\sqrt{\pi}} \lambda_{n}^{+}(B) \leq \mathrm{NS}_{t}\left(A_{t}\right)
$$

as $t \rightarrow 0$. On the other hand, if we let $\eta=\omega(1)$ in Theorem 1.1 increase sufficiently slowly, we see that for any sequence $A_{t}$, we can find $B_{t}$ with $\lambda_{1}^{+}\left(A_{t} \Delta B_{t}\right)=o(1)$ and

$$
\frac{2 \sqrt{t}}{\sqrt{\pi}} \lambda_{n}^{+}\left(B_{t}\right) \leq(1+o(1)) \mathrm{NS}_{t}\left(A_{t}\right)
$$

Theorems of this sort were introduced by Kearns and Ron 9 , and by Balcan et al. 4 in dimension 1, and extended to $\mathbb{T}^{n}$ by Kothari et al. 11. However, Kothari et al. gave a factor of $\kappa_{n}+\eta$ instead of $1+\eta$ on the right hand side, where $\kappa_{n}$ is an explicit constant that grows from 1 to $4 / \pi$ as $n$ goes from 1 to $\infty$. In fact, our analysis will be closely based on that of [11; our main contribution is an improved use of certain smoothness estimates, leading to an improved constant.

With (11) and Theorem 1.1 in hand, the algorithm for testing surface area is quite simple. By sampling $m$ pairs $(X, Y)$ according to the distribution above, one can estimate $\mathrm{NS}_{t}(A)$ to accuracy $O\left(m^{-1 / 2} \sqrt{\mathrm{NS}_{t}(A)}\right)$. Consider, then, the algorithm that says "yes" if and only if this estimate is smaller than $2 \sqrt{t / \pi}\left(S+O\left(m^{-1 / 2} t^{-1 / 4} S^{1 / 2}\right)\right)$. The completeness of the algorithm then follows immediately from (1) and Chebyshev's inequality.

The preceding algorithm is complete for any $m$ and $t$, but to show its soundness we will need to specify $m$ and $t$. First, we will assume that $m^{-1 / 2} t^{-1 / 4} S^{1 / 2} \leq \eta$. An application of Chebyshev's inequality then shows that for $A$ to be accepted with high probability, $A$ must satisfy $\mathrm{NS}_{t}(A) \leq 2 \sqrt{t / \pi}(1+$ $O(\eta)) S$. Applying Theorem 1.1 we see that there is some set $B$ with

$$
\lambda_{n}(A \Delta B) \leq O\left(S t^{1 / 2} \eta^{-1}\right)
$$

and

$$
\lambda_{n}^{+}(B) \leq \frac{\sqrt{\pi}}{2 \sqrt{t}}(1+o(\eta)) \mathrm{NS}_{t}(A) \leq(1+O(\eta)) S .
$$

Hence, the algorithm is sound provided that $S t^{1 / 2} \eta^{-1} \leq \epsilon$. Putting this condition together with the previous one, we see that $t=(\epsilon \eta / S)^{2}$ and $m=O\left(\eta^{-3} \epsilon^{-1} S^{2}\right)$ suffices. We summarize this discussion with the following algorithm.

Surface area testing in Gaussian and other spaces. Our analysis is not specific to the Lebesgue measure on the torus. For example, Theorem 1.1 also holds if $\lambda_{n}$ is replaced by the Gaussian measure and $\mathrm{NS}_{t}(A)$ is replaced by $\operatorname{Pr}\left(1_{A}(Z) \neq\right.$ $\left.1_{A}\left(\rho Z+\sqrt{1-\rho^{2}} Z^{\prime}\right)\right)$, where $Z$ and $Z^{\prime}$ are independent 


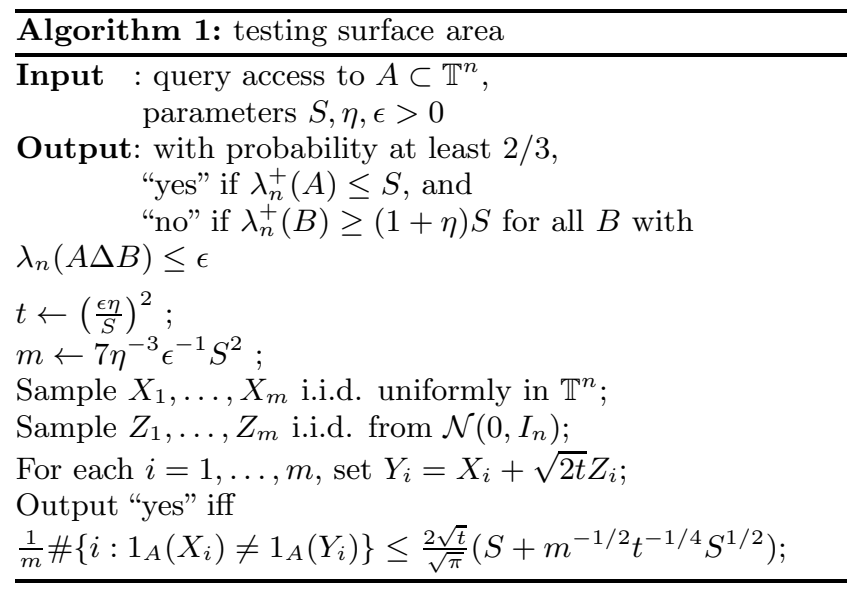

Gaussian vectors on $\mathbb{R}^{n}$. This Gaussian case was also considered in [11, who obtained the same result but with an extraneous factor of $4 / \pi$ on the right hand side. Since there is an analogue of (1) also in the Gaussian case (due to Ledoux [12]), one also obtains an algorithm for testing Gaussian surface area.

More generally, one could ask for a version of Theorem 1.1 on any weighted manifold. We propose a generalization of Theorem 1.1 in which the noise sensitivity is measured with respect to a Markov diffusion semigroup and the surface area is measured with respect to that semigroup's stationary measure. The class of stationary measures allowed by this extension includes log-concave measures on $\mathbb{R}^{n}$ and Riemannian volume elements on compact manifolds. Since Ledoux's argument 12] may be extended in this generality, our algorithm again extends.

\section{MARKOV SEMIGROUPS AND CURVA- TURE}

As stated in the introduction, we will carry out the proof of Theorem 1.1 in the setting of Markov diffusion semigroups. An introduction to this topic may be found in the Ledoux's monograph [13. To follow our proof, however, it is not necessary to know the general theory; we will be concrete about the Gaussian and Lebesgue cases, and it is enough to keep one of these in mind.

Let $(M, g)$ be a smooth, Riemannian $n$-manifold and consider the differential operator $L$ that is locally defined by

$$
(L f)(x)=\sum_{i, j=1}^{n} g^{i j}(x) \frac{\partial^{2} f}{\partial x_{i} \partial x_{j}}+\sum_{i=1}^{n} b^{i}(x) \frac{\partial f}{\partial x_{i}}
$$

where $b^{i}$ are smooth functions and $\left(g^{i j}(x)\right)_{i, j=1}^{n}$ is the inverse tensor of $g$ in local coordinates. Such an operator induces a semigroup $\left(P_{t}\right)_{t \geq 0}$ of operators which satisfies $\frac{d}{d t} P_{t} f=L f$. There are certain technical issues, which we will gloss over here, regarding the domains of these operators. We will assume that the domain of $L$ contains an algebra $\mathcal{A}$ satisfying $P_{t} \mathcal{A} \subset \mathcal{A}$. We will assume moreover that $P_{t}$ has an invariant probability distribution $\mu$ which is absolutely continuous with respect to the Riemannian volume element on $M$; we will also assume that $\mathcal{A}$ is dense in $L_{p}(\mu)$ for every $\mu$. In any case, these assumptions are satisfied in many interest- ing examples, such as when $P_{t}$ is the heat semigroup on a compact Riemannian manifold, or when $P_{t}$ is the Markov semigroup associated with a log-concave measure $\mu$ on $\mathbb{R}^{n}$. See 13 for more details.

Given a Markov semigroup $P_{t}$, we define the noise sensitivity by

$$
\mathrm{NS}_{t}(A)=\int_{M}\left|P_{t} 1_{A}(x)-1_{A}(x)\right| d \mu(x) .
$$

The probabilistic interpretation of this quantity is given by the Markov process associated with $P_{t}$. This is a Markov process $\left(X_{t}\right)_{t \in \mathbb{R}}$ with the property that for any $f \in L_{1}(\mu)$, $\mathbb{E}\left(f\left(X_{t}\right) \mid X_{0}\right)=\left(P_{t} f\right)\left(X_{0}\right)$. Given such a process, the noise sensitivity may be alternatively written as $\mathrm{NS}_{t}(A)=$ $\operatorname{Pr}\left(1_{A}\left(X_{0}\right) \neq 1_{A}\left(X_{t}\right)\right)$.

The other notion we need is that of surface area. Recalling that $\mu$ was assumed to have a density with respect to the Riemannian volume, we define

$$
\mu^{+}(A)=\int_{\partial A} \mu(x) d \mathcal{H}_{n-1}(x),
$$

where $\mathcal{H}_{n-1}$ is the $(n-1)$-dimensional Hausdorff measure.

Let us revisit $\left(\mathbb{T}^{n}, \lambda_{n}\right)$ and $\left(\mathbb{R}^{n}, \gamma_{n}\right)$ in our more abstract setting. In the case of $\mathbb{T}^{n}$, we set $L$ to be $\sum_{i=1}^{n} \frac{\partial^{2}}{\partial x_{i}^{2}}$. Then $P_{t}$ is given by

$$
\left(P_{t} f\right)(x)=\int_{\mathbb{R}^{n}} f(x+\sqrt{2 t} y) d \gamma_{n}(y)
$$

The associated Markov process $X_{t}$ is simply Brownian motion, and so we see that the noise sensitivity defined in (3) coincides with the definition that we gave in the introduction.

In the Gaussian case, we define $L$ by

$$
(L f)(x)=\sum_{i=1}^{n}\left(\frac{\partial^{2} f}{\partial x_{i}^{2}}-x_{i} \frac{\partial f}{\partial x_{i}}\right) .
$$

Then $P_{t}$ is given by

$$
\left(P_{t} f\right)(x)=\int_{\mathbb{R}^{n}} f\left(e^{-t} x+\sqrt{1-e^{-2 t}} y\right) d \gamma_{n}(y) .
$$

The associated Markov process $X_{t}$ is the Ornstein-Uhlenbeck process, which is the Gaussian process for which $\mathbb{E} X_{s}^{T} X_{t}=$ $e^{-|s-t|} I_{n}$.

In order to state our generalization of Theorem 1.1, we need a geometric condition on the semigroup $P_{t}$. Following Bakry and Emery [2] (see also [13]), we say that the semigroup $\left(P_{t}\right)_{t \geq 0}$ has curvature $R$ if the inequality $\left|\nabla P_{t} f\right| \leq$ $e^{-R t} P_{t}|\nabla f|$ holds pointwise for every $f \in \mathcal{A}$. One can check easily from the definitions that in the heat semigroup on $\mathbb{T}^{n}$ has curvature 0 , while the Ornstein-Uhlenbeck semigroup on $\mathbb{R}^{n}$ has curvature 1 .

In this abstract setting, our main theorem is the following. Observe that by specializing to the torus $\mathbb{T}^{n}$ with the Lebesgue measure $\lambda_{n}$ and curvature $R=0$, we recover Theorem 1.1 . 
TheOREm 2.1. Suppose that $P_{t}$ has curvature $R$. For any $A \subset M$ with $\mathcal{C}^{1}$ boundary and for every $\eta, t>0$, there is a set $B \subset M$ with $\mu(A \Delta B) \leq \mathrm{NS}_{t}(A) / \eta$ and

$$
\mu_{n}^{+}(B) \leq \sqrt{\frac{\pi}{2}}\left(1+\frac{\sqrt{\pi} \eta}{\sqrt{\log (1 / \eta)}}\left(1+o_{\eta}(1)\right)\right) c_{R}(t) \mathrm{NS}_{t}(A)
$$

where $c_{R}(t)=\left(\frac{e^{2 R t}-1}{R}\right)^{-1 / 2}$ if $R \neq 0$ and $c_{0}(t)=(2 t)^{-1 / 2}$. Here, $o_{\eta}(1)$ denotes a quantity that tends to zero as $\eta \rightarrow 0$.

In order to prove Theorem 2.1 we will construct the set $B$ in a randomized way, using a construction that is due to Kothari et al. 11. Their construction is quite simple: we first smooth the function $1_{A}$ using $P_{t}$ and then threshold $P_{t} 1_{A}$ to obtain $1_{B}$. One difficulty with this procedure is to find a suitable threshold value. Kothari et al. dealt with this difficulty in a remarkably elegant way: they showed that after thresholding at an appropriately chosen random value, the expected surface area of the resulting set is small. In particular, there is some threshold value that suffices.

The analysis of the random thresholding procedure uses two main tools: the first is the coarea formula (see, e.g. [8]), which will allow us to express the expected surface area of our thresholded set in terms of the gradient of $P_{t} 1_{A}$.

Theorem 2.2 (CoArea formula). For any $\mathcal{C}^{1}$ function $f: M \rightarrow[0,1]$, any $\mu \in L_{1}(M)$, and any $\psi \in L_{\infty}([0,1])$,

$$
\begin{aligned}
\int_{0}^{1} \psi(s) \int_{\{x \in M: f(x)=s\}} \mu(x) d \mathcal{H}_{n-1}(x) d s \\
=\int_{M} \psi(f(x))|\nabla f(x)| \mu(x) d x
\end{aligned}
$$

Our second tool is a pointwise bound on $\left|\nabla P_{t} f\right|$ for any $f: M \rightarrow[0,1]$. This will allow us, after applying the coarea formula, to obtain a sharp bound on the integral involving $\left|\nabla P_{t} 1_{A}\right|$.

Theorem 2.3 [3]). If $P_{t}$ has curvature $R$ then for any $f: M \rightarrow[0,1]$ and any $t>0$,

$$
\left|\nabla P_{t} f\right| \leq c_{R}(t) I\left(P_{t} f\right)
$$

where $c_{R}(t)=\left(\frac{e^{2 R t}-1}{R}\right)^{-1 / 2}$ if $R \neq 0$ and $c_{0}(t)=(2 t)^{-1 / 2}$.

For $g: M \rightarrow[0,1]$, let $g^{\geq s}$ denote the set $\{x \in M: g(x) \geq$ $s\}$. If $g$ is continuous then $\partial g^{\geq s}=\{x \in M: g(x)=s\}$. Hence the surface area of $g^{\geq s}$ is simply

$$
\mu^{+}\left(g^{\geq s}\right)=\int_{\{x \in M: g(x)=s\}} \mu(x) d \mathcal{H}_{n-1}(x),
$$

and so the coarea formula (Theorem 2.2) implies that

$\int_{0}^{1} \psi(s) \mu^{+}\left(g^{\geq s}\right) d s=\int_{M} \psi(g(x))|\nabla g(x)| \mu(x) d x=\mathbb{E} \psi(g)|\nabla g|$.

(From here on, we will often write $\mathbb{E}$ for the integral with respect to $\mu$ which, recall, is a probability measure.) On the other hand, $\int_{0}^{1} \psi(s) \mu^{+}\left(g^{\geq s}\right) d s \geq \min _{s \in[0,1]} \mu^{+}\left(g^{\geq s}\right) \int_{0}^{1} \psi(s) d s$. In particular, if we can show that $\mathbb{E} \psi(g)|\nabla g|$ is small then it will follow that $\mu^{+}\left(g^{\geq s}\right)$ is small for some $s$.

Unsurprisingly, the quantity $\mathbb{E} \psi(g)|\nabla g|$ is quite sensitive to the choice of $\psi$. In order to get the optimal constant in Theorem 2.1 we need to choose a particular function $\psi$. Namely, we define

$$
\psi(s)=\frac{\frac{1}{2}-\left|s-\frac{1}{2}\right|}{I(s)} .
$$

Lemma 2.4. For any measurable $A \subset M$ and any $t>0$,

$$
\mathbb{E} \psi\left(P_{t} 1_{A}\right)\left|\nabla P_{t} 1_{A}\right| \leq c_{R}(t) \mathrm{NS}_{t}(A) .
$$

Proof. By Theorem 2.3

$$
\begin{aligned}
\mathbb{E} \psi\left(P_{t} 1_{A}\right)\left|\nabla P_{t} 1_{A}\right| & \leq c_{R}(t) \mathbb{E} \psi\left(P_{t} 1_{A}\right) I\left(P_{t} 1_{A}\right) \\
& =c_{R}(t) \mathbb{E}\left(\frac{1}{2}-\left|P_{t} 1_{A}-\frac{1}{2}\right|\right) .
\end{aligned}
$$

Now, $\frac{1}{2}-\left|x-\frac{1}{2}\right|=\min \{x, 1-x\}$ and so

$$
\begin{aligned}
\mathbb{E}\left(\frac{1}{2}-\left|P_{t} 1_{A}-\frac{1}{2}\right|\right) & =\mathbb{E} \min \left\{P_{t} 1_{A}, 1-P_{t} 1_{A}\right\} \\
& \leq \mathbb{E}\left|P_{t} 1_{A}-1_{A}\right| \\
& =\operatorname{NS}_{t}(A) .
\end{aligned}
$$

Going back to the discussion before Lemma 2.4 we have shown that

$$
\begin{aligned}
\min _{s \in[0,1]} \mu^{+}\left(\left(P_{t} 1_{A}\right)^{\geq s}\right) \int_{0}^{1} \psi(s) d s & \leq \int_{0}^{1} \mu^{+}\left(\left(P_{t} 1_{A}\right)^{\geq s}\right) \psi(s) d s \\
& \leq c_{R}(t) \operatorname{NS}_{t}(A) .
\end{aligned}
$$

Since we are concerned in this work with optimal constants, let us compute $\int_{0}^{1} \psi(s) d s$ :

$$
\text { LEMma 2.5. } \int_{0}^{1} \psi(s) d s=\sqrt{\frac{2}{\pi}} \text {. }
$$

Proof. We use the substitution $s=\Phi(y)$. Then $d s=$ $\phi(y) d y$ and $I(s)=\phi(y)$. Hence,

$\int_{0}^{1} \psi(s) d s=\int_{-\infty}^{\infty} \frac{1}{2}-\left|\frac{1}{2}-\Phi(y)\right| d y=2 \int_{0}^{\infty} 1-\Phi(y) d y$,

where the last equality follows because $\Phi(-t)=1-\Phi(t)$ and $\Phi(t) \geq \frac{1}{2}$ for $t \geq 0$. Recalling the definition of $\Phi$, if we set $Z$ to be a standard Gaussian variable then

$$
2 \int_{0}^{\infty} 1-\Phi(y) d y=2 \mathbb{E} \max \{0, Z\}=\mathbb{E}|Z|=\sqrt{\frac{2}{\pi}} .
$$

Combining Lemmas 2.5 and 2.4 we have shown the existence of some $s \in[0,1]$ such that $\mu^{+}\left(\left(P_{t} 1_{A}\right)^{\geq s}\right) \leq \sqrt{\pi / 2} c_{R}(t) \mathrm{NS}_{t}(A)$. This is not quite enough to prove Theorem 2.1 because we 
need to produce a set $B$ such that $\mu(B \Delta A)$ is small. In general, $\left(P_{t} 1_{A}\right)^{\geq s}$ may not be close to $A$; however, if $s \in[\eta, 1-\eta]$ then they are close:

Lemma 2.6. For any $t>0$, if $s \in[\eta, 1-\eta]$ then

$$
\mu\left(\left(P_{t} 1_{A}\right)^{\geq s} \Delta A\right) \leq \frac{1}{\eta} \mathrm{NS}_{t}(A) .
$$

Proof. Note that if the indicator of $\left(P_{t} 1_{A}\right)^{\geq s}$ is not equal to $1_{A}$ then either $1_{A}=0$ and $P_{t} 1_{A} \geq s$ or $1_{A}=1$ and $P_{t} 1_{A}<s$. If $s \in[\eta, 1-\eta]$ then either case implies that $\left|P_{t} 1_{A}-1_{A}\right| \geq \eta$. Hence,

$$
\mu\left(\left(P_{t} 1_{A}\right)^{\geq s} \Delta A\right) \leq \frac{1}{\eta} \mathbb{E}\left|P_{t} 1_{A}-1_{A}\right|=\frac{1}{\eta} \mathrm{NS}_{t}(A) .
$$

To complete the proof of Theorem 2.1 we need to invoke Lemmas 2.4 and 2.5 in a slightly different way from before. Indeed, with Lemma 2.6 in mind we want to show that there is some $s$ for which $\mu^{+}\left(\left(P_{t} 1_{A}\right)^{+}\right)$is small and such that $s$ is not too close to zero or one. For this, we note that

$$
\begin{aligned}
\int_{\eta}^{1-\eta} \psi(s) d s \min _{s \in[\eta, 1-\eta]} \mu^{+}\left(g^{\geq s}\right) & \leq \int_{\eta}^{1-\eta} \psi(s) \mu^{+}\left(g^{\geq s}\right) d s \\
& \leq \int_{0}^{1} \psi(s) \mu^{+}\left(g^{\geq s}\right) d s
\end{aligned}
$$

With $g=P_{t} 1_{A}$, we see from Lemma 2.4 that

$$
\min _{s \in[\eta, 1-\eta]} \mu^{+}\left(\left(P_{t} 1_{A}\right)^{\geq s}\right) \leq \frac{c_{R}(t) \mathrm{NS}_{t}(A)}{\int_{\eta}^{1-\eta} \psi(s) d s} .
$$

To compute the denominator, one checks (see, e.g., 3]) the limit $I(x) \sim x \sqrt{2 \log (1 / x)}$ as $x \rightarrow 0$ and so $\psi(x) \sim(2 \log (1 / x))^{-1 / 2}$ as $x \rightarrow 0$. Hence, $\int_{0}^{\eta} \psi(s) d s \sim \eta(2 \log (1 / \eta))^{-1 / 2}$ as $\eta \rightarrow 0$ and since $\psi(x)$ is symmetric around $x=1 / 2$,

$$
\int_{\eta}^{1-\eta} \psi(s) d s=\int_{0}^{1} \psi(s) d s-\frac{\sqrt{2} \eta}{\sqrt{\log (1 / \eta)}}(1+o(1))
$$

as $\eta \rightarrow 0$. Applying this to (4) (along with the formula from Lemma 2.5), there must exist some $s \in[\eta, 1-\eta]$ with

$$
\begin{aligned}
\mu^{+}\left(\left(P_{t} 1_{A}\right)^{\geq s}\right) & \leq \frac{c_{R}(t) \mathrm{NS}_{t}(A)}{\int_{\eta}^{1-\eta} \psi(s) d s} \\
& \leq \sqrt{\frac{\pi}{2}}\left(1+\frac{\sqrt{\pi} \eta}{\sqrt{\log (1 / \eta)}}(1+o(1))\right) c_{R}(t) \mathrm{NS}_{t}(A)
\end{aligned}
$$

Taking $B=\left(P_{t} 1_{A}\right)^{\geq s}$ for such an $s$, we see from Lemma 2.6 that this $B$ satisfies the claim of Theorem 2.1, thereby completing the proof of that theorem.

\section{ACKNOWLEDGEMENTS}

We would like to thank E. Mossel for helpful discussions, and M. Ledoux for pointing out that the argument of 12 may be extended. Much of the work on this paper was done while the author was visiting the Simons Institute for the Theory of Computing, which he thanks for its hospitality.

The author is partially supported by DTRA grant HDTRA113-1-0024.

\section{REFERENCES}

[1] AJ Baddeley, Hans-Jørgen G Gundersen, and Luis Manuel Cruz-Orive. Estimation of surface area from vertical sections. Journal of Microscopy, 142(3):259-276, 1986.

[2] D. Bakry and M. Émery. Diffusions hypercontractives. Séminaire de Probabilités XIX 1983/84, pages 177-206, 1985.

[3] D. Bakry and M. Ledoux. Lévy-Gromov's isoperimetric inequality for an infinite dimensional diffusion generator. Inventiones mathematicae, 123(2):259-281, 1996.

[4] Maria-Florina Balcan, Eric Blais, Avrim Blum, and Liu Yang. Active property testing. In Proceedings of the 2012 IEEE 53rd Annual Symposium on Foundations of Computer Science, FOCS '12, pages 21-30, Washington, DC, USA, 2012. IEEE Computer Society.

[5] Mikhail Belkin, Hariharan Narayanan, and Partha Niyogi. Heat flow and a faster algorithm to compute the surface area of a convex body. In FOCS'06, pages 47-56. IEEE, 2006.

[6] Manuel Blum, Michael Luby, and Ronitt Rubinfeld. Self-testing/correcting with applications to numerical problems. Journal of computer and system sciences, 47(3):549-595, 1993.

[7] Antonio Cuevas, Ricardo Fraiman, and Alberto Rodríguez-Casal. A nonparametric approach to the estimation of lengths and surface areas. The Annals of Statistics, 35(3):1031-1051, 2007.

[8] Herbert Federer. Geometric measure theory. Springer-Verlag, 1969.

[9] Michael Kearns and Dana Ron. Testing problems with sublearning sample complexity. In Proceedings of the 11th annual Conference on Learning Theory, pages 268-279, 1998.

[10] Adam R. Klivans, Ryan O'Donnell, and Rocco A. Servedio. Learning geometric concepts via gaussian surface area. In FOCS'08, pages 541-550. IEEE, 2008.

[11] P. Kothari, A. Nayyeri, R. O'Donnell, and C. Wu. Testing surface area. In Proceedings of the Twenty-Fifth Annual ACM-SIAM Symposium on Discrete Algorithms, pages 1204-1214.

[12] M. Ledoux. Semigroup proofs of the isoperimetric inequality in Euclidean and Gauss space. Bulletin des sciences mathématiques, 118(6):485-510, 1994.

[13] Michel Ledoux. The geometry of Markov diffusion generators. Ann. Fac. Sci. Toulouse Math. (6), 9(2):305-366, 2000. Probability theory.

[14] Yu-Shen Liu, Jing Yi, Hu Zhang, Guo-Qin Zheng, and Jean-Claude Paul. Surface area estimation of digitized 3d objects using quasi-monte carlo methods. Pattern Recognition, 43(11):3900-3909, 2010.

[15] Luis Antonio Santaló. Introduction to integral geometry. Hermann, 1953. 